\title{
Radiation-induced alternative transcripts as detected in total and polysome-bound mRNA
}

\author{
Amy Wahba ${ }^{1}$, Michael C. Ryan ${ }^{2}$, Uma T. Shankavaram ${ }^{1}$, Kevin Camphausen ${ }^{1}$ and \\ Philip J. Tofilon ${ }^{1}$ \\ ${ }^{1}$ Radiation Oncology Branch, National Cancer Institute, Bethesda, MD 20892, USA \\ ${ }^{2}$ In Silico Solutions, Falls Church, VA 22043, USA \\ Correspondence to: Philip J. Tofilon, email: philip.tofilon@nih.gov \\ Keywords: radiation; polysomes; alternative splicing; gene expression \\ Received: August 09, $2017 \quad$ Accepted: September 16, $2017 \quad$ Published: October 09, 2017 \\ Copyright: Wahba et al. This is an open-access article distributed under the terms of the Creative Commons Attribution License \\ 3.0 (CC BY 3.0), which permits unrestricted use, distribution, and reproduction in any medium, provided the original author and \\ source are credited.
}

\section{ABSTRACT}

Alternative splicing is a critical event in the posttranscriptional regulation of gene expression. To investigate whether this process influences radiation-induced gene expression we defined the effects of ionizing radiation on the generation of alternative transcripts in total cellular mRNA (the transcriptome) and polysome-bound mRNA (the translatome) of the human glioblastoma stem-like cell line NSC11. For these studies, RNA-Seq profiles from control and irradiated cells were compared using the program Spliceseq to identify transcripts and splice variations induced by radiation. As compared to the transcriptome (total RNA) of untreated cells, the radiation-induced transcriptome contained 92 splice events suggesting that radiation induced alternative splicing. As compared to the translatome (polysome-bound RNA) of untreated cells, the radiation-induced translatome contained 280 splice events of which only 24 were overlapping with the radiation-induced transcriptome. These results suggest that radiation not only modifies alternative splicing of precursor mRNA, but also results in the selective association of existing mRNA isoforms with polysomes. Comparison of radiation-induced alternative transcripts to radiation-induced gene expression in total RNA revealed little overlap (about 3\%). In contrast, in the radiation-induced translatome, about $38 \%$ of the induced alternative transcripts corresponded to genes whose expression level was affected in the translatome. This study suggests that whereas radiation induces alternate splicing, the alternative transcripts present at the time of irradiation may play a role in the radiation-induced translational control of gene expression and thus cellular radioresponse.

\section{INTRODUCTION}

The development of molecularly targeted approaches for enhancing the efficacy of radiotherapy, a primary treatment modality for most solid tumors, requires a comprehensive understanding of the processes comprising cellular radioresponse. Along these lines, fundamental determinants of radioresponse, such as DNA repair and cell cycle checkpoint activation, have been best described in terms of the post-translational modification of existing proteins. Modifications in gene expression, however, are also a consequence of irradiation suggesting a role for de novo protein synthesis in determining cellular radioresponse. Towards understanding the biological significance of radiation-induced gene expression, studies initially focused on defining the changes in the transcriptome (total cellular mRNA) in irradiated cells [1-6]. However, interpreting the significance of such transcriptome analyses is complicated by the lack of correlation between the radiation-induced changes in mRNA and their corresponding proteins [7]. To address the discrepancy between the radiation-induced transcriptome and proteome, polysome-bound RNA, which identifies 
genes undergoing translation and defines the translatome, was subjected to microarray analysis and compared to the traditional microarray approach using total cellular mRNA $[8,9]$. The results of these studies showed that the number of genes in the radiation-induced translatome was substantially greater than those in the radiation-induced transcriptome and that there was little to no overlap between the two expression profiles. Moreover, in contrast to the changes in the transcriptome after irradiation, there was a strong correlation between genes appearing in the radiation induced translatome and changes in the levels of their corresponding proteins. Thus, data indicate that radiation modifies gene expression primarily through translational control.

One of the critical events in the post-transcriptional regulation of gene expression is the splicing of introns from precursor mRNA (pre-mRNA) [10]. Splicing can be the same in each pre-mRNA from a given gene or can proceed through alternative splicing events resulting in the generation of mRNA isoforms (alternative transcripts) from the same genomic sequence. Most human genes undergo alternative splicing (AS), which is recognized as a major source of protein diversity [11-13]. A wellestablished mechanistic consequence of $\mathrm{AS}$ is the formation of transcripts with increased susceptibility to nonsense mediated decay, which ultimately reduces gene expression [12-14]. However, in HEK293T cells and in mouse embryonic stem cells the association of mRNAs with polysomes has been reported to occur in an isoform specific manner $[15,16]$, suggesting a role for AS in the translational control of gene expression.

Radiation has been shown to induce AS in the evaluation of individual genes [17-19] and at the whole transcriptome level using exon arrays [20-22]. Whether AS plays a role in radiation-induced translational control of gene expression has not yet been reported. In the study described here we used RNA-Seq and the bioinformatics program SpliceSeq to identify alternative transcripts in the transcriptome and translatome after irradiation of a glioblastoma stem-like cell line. Data presented here show that radiation induced substantially more AS events in the polysome fraction (translatome) as compared to total cellular RNA (transcriptome) with only a minor overlap between the 2 fractions. Moreover, many of the genes corresponding to splice events unique to the radiation-induced translatome were associated with DNA repair processes. These results suggest that radiation not only induces AS, but that there is also a preferential recruitment of specific mRNA isoforms to polysomes after irradiation, suggesting a role for AS in the radiationinduced translational control of gene expression.

\section{RESULTS}

We recently described the radiation-induced transcriptomes (total cellular mRNA) and translatomes (polysome-bound mRNA) for the NSC11 glioblastoma stem-like cell (GSC) line along with 2 additional GSC lines using microarray analysis [9]. In that study, radiation-induced changes in polysome-bound mRNA correlated with changes in corresponding proteins and associated cell processes supporting the concept that radiation-induced translational control of gene expression is a fundamental component of radioresponse. To build on these data and as an initial investigation into the potential role of alternative splicing (AS) in the radiation-induced translational control of gene expression, we used RNA-Seq and SpliceSeq analyses on untreated and irradiated NSC11 cells. Rather than using a single parameter such as RPKM (gene reads per kilobase of transcript per million aligned reads), identification of significant AS events using SpliceSeq was based on the combination of 5 criteria (as listed in Methods), which included the necessity of being detected in 5 of the 6 biological replicates.

\section{Alternative splice events specific to polysome- bound RNA in untreated cells}

Initially, we compared the alternative transcript diversity in polysome-bound versus total RNA from untreated NSC11 cells. Specifically, from both polysomebound RNA (isolated by sucrose gradient fractionation) or total cellular RNA, polyA ${ }^{+}$selected RNA was subjected to paired-end sequencing with mapping to the human hg19 reference genome (see Supplementary Table 1 for RNASeq parameters); we then used the comparison option of SpliceSeq to identify genes with splicing differences between polysome-bound mRNA (the translatome) and total cellular mRNA (the transcriptome). Comparison of mRNA isoforms detected in polysome-bound mRNA to those in total mRNA revealed 666 splicing events significantly enriched in the polysome-bound RNA (Figure 1A) corresponding to 567 genes (Supplementary Table 2). The splice events specific to polysomes divided into 7 types with more than half attributed to mRNA with alternatively retained introns (RI) and mRNA that had alternative exon skip (ES) events and about 20\% that had an alternative termination (AT). The splice events specific to polysome-bound mRNA primarily involved one splice event per gene (simple events) with most of the affected genes containing only a single event, suggesting that the single splice event was sufficient to change the polysome association of most of the identified transcripts in untreated samples (Figure 1B). Genes containing more than one splice event per gene (complex) comprised only $13.6 \%$ of the genes undergoing alternative splicing. The selective association of alternative transcripts with polysomes suggests that AS influences RNA translation in NSC11 cells, consistent with results obtained from other cell lines $[15,16]$. 


\section{Radiation-induced alternative transcripts}

To investigate the effects of radiation on AS, total cellular mRNA or polysome-bound mRNA was collected from NSC11 cells $1 \mathrm{~h}$ after irradiation (2 Gy) or after mock irradiation (control) and subjected to RNA-Seq. An advantage provided by using NSC11 cells is that the majority of changes in the translatome occur at $1 \mathrm{~h}$ after irradiation [9], which reduces the potential contribution of transcriptional modifications allowing for a more direct investigation of the role of AS in the translational control of gene expression [23]. For each mRNA compartment, the comparison option of SpliceSeq was used to identify genes with splicing differences between control and irradiated cells. Splice events enriched in total cellular mRNA or polysome-bound mRNA after irradiation are shown in Figure 2A. Ninety-two splice events were detected in the radiation-induced transcriptome (total RNA), which, consistent with previous studies using exon arrays $[20,21]$, suggests that radiation induces AS of premRNA. However, 280 splice events were detected in the radiation-induced translatome (polysome-bound RNA) of which only 24 were overlapping with the radiationinduced transcriptome. Given the discrepancy between radiation-induced splice events in the translatome and transcriptome, it appears that radiation not only modifies AS of pre-mRNA, but also results in the selective association of existing mRNA isoforms with polysomes. The types of radiation-induced splice events appeared to be similar between the translatome and transcriptome with more than half being of the exon skipping (ES) form in each compartment (Figure 2B and 2C). The vast

A

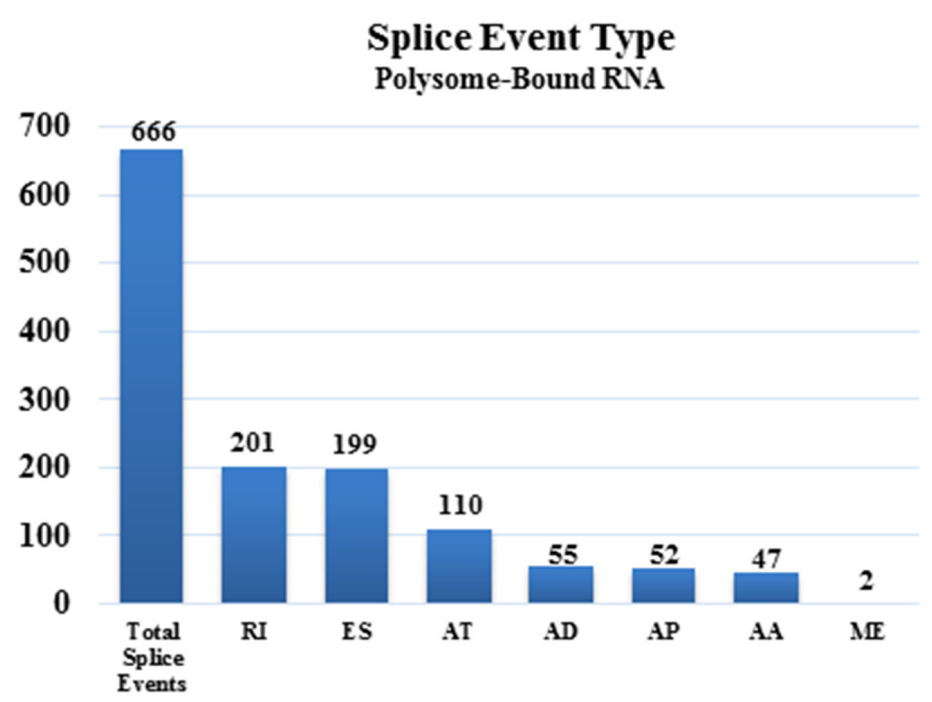

B

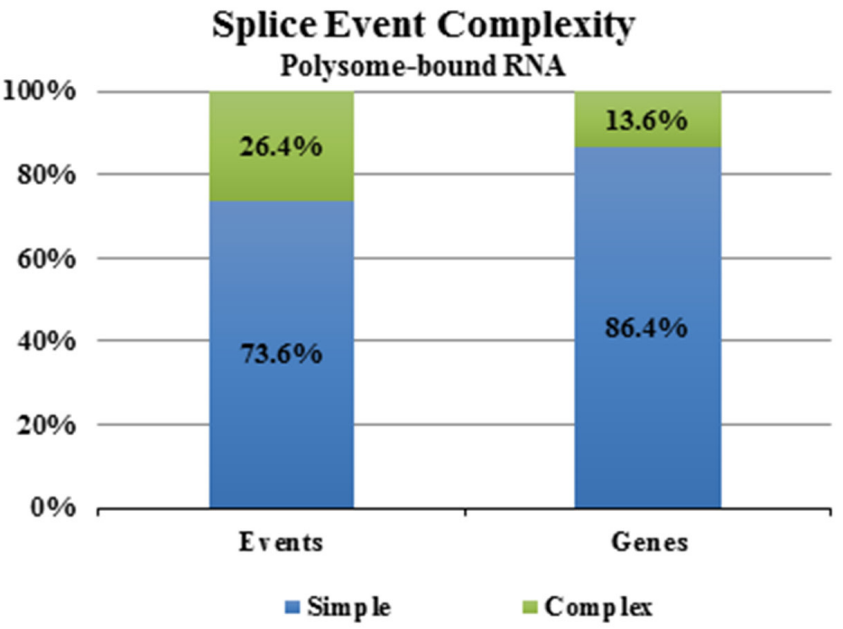

Figure 1: Alternative splice events in the translatome as compared to the transcriptome. (A) Numbers of significant alternative splice events in the translatome of NSC11 as compared to the transcriptome according to category [alternate acceptor (AA), alternate donor (AD), alternate promoter (AP), alternate terminator (AT), exon skip (ES), mutually exclusive exons (ME), retained intron (RI)]. (B) Complexity of splice events in polysome-bound RNA as compared to total RNA. Simple (blue) refers to 1 event and complex (green) refers to more than 1 event. 
majority of splice events induced by radiation in both the transcriptome and translatome analyses were classified as simple events (one splice event per gene) with most of the affected genes containing only one event (Figure 2D and $2 \mathrm{E})$. These results suggest that a single splicing event is sufficient to affect the radiation-mediated enrichment of an mRNA in the polysome-bound compartment.

\section{Validation of splice events}

The presence of the radiation-induced splice events was validated using qRT-PCR. The 6 genes evaluated involved radiation-induced splice events enriched in polysome-associated RNA only (UBE2G2, eIF4H), total cellular RNA only (STRN3, NDEL1), and splice events common to both (TGFBR2, SOS1). These genes were selected for evaluation because they contained among the largest RPKMs for alternative exon skip events, the most common radiation-induced splice event detected in the transcriptome and translatome; were predicted to be modified in only the translatome, only the transcriptome or both and for which there were commercially available primer assays. Primers were selected based on sequence analysis to flank a splice junction of the transcript as predicted by SpliceSeq. This allowed detection of the changes in each transcript predicted to be alternatively spliced after irradiation and thus to reflect changes in the splice event rather than transcript abundance. The dPSI (change in percent spliced in and a measure of splice frequency) for each of these splice events in the individual replicates from each treatment condition show the change in splicing after irradiation as well as a high reproducibility across the 6 replicates (Figure 3A). The qRT-PCR analysis (Figure 3B) indicates the same qualitative changes induced by radiation as detected by RNA-Seq/SpliceSeq with the spliced exon of UBE2G2 reduced in polysomebound RNA after irradiation compared to a constant value in total RNA after irradiation. The spliced exon in eIF4H increased in the polysome fraction after irradiation and in NDEL1 increased in the total RNA fraction after irradiation, both consistent with the RNA-Seq/SpliceSeq data. As predicted, the spliced exon in STRN3 increased in the total RNA fraction in response to radiation and in TGFBR2 and SOS1, the spliced exon increased in both polysome-bound RNA and total RNA. Thus, the qRTPCR results validate the RNA-Seq/SpliceSeq analyses of radiation-induced alternate transcripts in total and polysome-bound mRNA. To determine whether the splice events induced by radiation were specific to NSC11 cells, qRT-PCR analysis was performed using the GSC line, 0923. As shown in Figure 3C, for 3 of the 6 genes evaluated (TGFBR2, STRN3 and SOS1), the radiationinduced alternative transcripts were similar for NSC11 and 0923 cells. For polysome-bound UBE2G2, a change was detected in 0923 but in the opposite direction as that in NSC11. For eIF4H and NDEL1, whereas the changes in the radiation-induced splice events in the polysome fraction were similar between the 2 cell lines, the relative changes detected in the transcriptome (total RNA) were slightly different. Specifically, the eIF4H splice event was significantly increased in 0923 cells, whereas in NSC11 it only trended towards an increase and the NDEL1 splice event was significantly increased in irradiated NSC11 cells but only trended toward an increase in 0923. These results suggest that at least some radiation-induced changes in AS are not unique to NSC11 cells.

\section{Functional analysis of alternative transcripts detected after irradiation}

The radiation-induced splice events in total cellular RNA (92) mapped to 89 genes and the radiation-induced splice events in polysome-bound RNA (280) mapped to 262 genes (Supplementary Tables 3 and 4). To generate insight into the functional consequences of the radiationinduced alternative transcripts, the gene lists from the total RNA and polysome-bound RNA were uploaded to Ingenuity Pathway Analysis (IPA), which distributes genes into curated networks and associates the networks with cell functions as well as canonical pathways. As shown in Figure 4A, the genes containing the radiation-induced splice events in total RNA distributed to six canonical pathways, none of which were significant at $\mathrm{p}<0.01$ $(-\log (\mathrm{p}$-value $) \geq 2)$. However, the genes containing the radiation-induced splice events in polysome-bound RNA contained 10 canonical pathways, 4 of which were significant $(\mathrm{p}<0.01)$ : DNA Double-Strand Break Repair by Homologous Recombination, Ceramide Degradation, Sphingosine and Sphingosine-1-phosphate Metabolism and L-carnitine Biosynthesis. The networks enriched in total RNA and polysome-bound RNA after irradiation are shown in Tables 1 and 2, respectively. While 9 networks were identified in total RNA, 22 were found in polysomebound RNA including "DNA Replication, Recombination, and Repair", processes critical in determining cellular radioresponse. This network, which is diagramed in Figure 4B, has 17 subgroups with increased functional specificity (unfilled symbols). In polysome-bound RNA there were 22 genes changed after irradiation (grey symbols, Figure 4B) that map to these specific functions; 3 of these genes (highlighted in red) were also affected at the level of total RNA. These data suggest that the radiation-induced alternative transcripts detected in the polysome bound RNA may contribute to DNA damage response after irradiation.

\section{Relationship between radiation-induced AS and gene expression}

Because RNA-Seq data allows for not only evaluation of alternative transcripts (SpliceSeq), but also changes in gene expression, we compared radiation- 
induced alternative transcripts to radiation-induced gene expression as detected in total RNA and polysome-bound RNA. In terms of the radiation-induced gene expression substantially more genes were affected by radiation in the translatome (3929) versus the transcriptome (284) (Figure 5 ), consistent with previous reports based on microarray analysis [8, 9, 24]. In the transcriptome (Figure 5A), comparison of radiation-induced alternative transcripts to gene expression showed little overlap with about $3 \%$ of alternative transcripts also being reflected as changes at the gene level. These data suggest that radiation-induced AS of pre-mRNA does not, for the most part, play a role in determining the radiation-induced transcriptome at $1 \mathrm{~h}$. However, in the radiation-induced translatome, about $38 \%$ of the induced alternative transcripts corresponded to genes whose polysome-association was affected after irradiation (Figure 5B). These data suggest that a portion of the radiation-induced AT enriched in the polysomebound RNA may directly influence the translational efficiency of the corresponding gene. Functional analysis of the radiation-induced AT detected in the transcriptome and translatome are shown in Figure 4. Regarding the gene expression changes induced in the transcriptome and translatome at $1 \mathrm{~h}$ after irradiation, results from IPA are shown in Tables 3 and 4 with the molecules in the top 10 networks in the transcriptome and in the translatome listed in Supplementary Tables 5 and 6, respectively. Analyses of the radiation-induced gene expression in total RNA (Table 3 ) and polysome-bound RNA (Table 4) indicate that networks associated with DNA replication, recombination, and repair, are enriched in both the transcriptome and the translatome.

\section{DISCUSSION}

In the study reported here we used RNA-Seq and the mRNA isoform identification tool SpliceSeq to investigate the contribution of AS to radiation-induced gene expression. At the whole genome level, the effects of radiation on AS have previously been reported for human lymphoblasts,
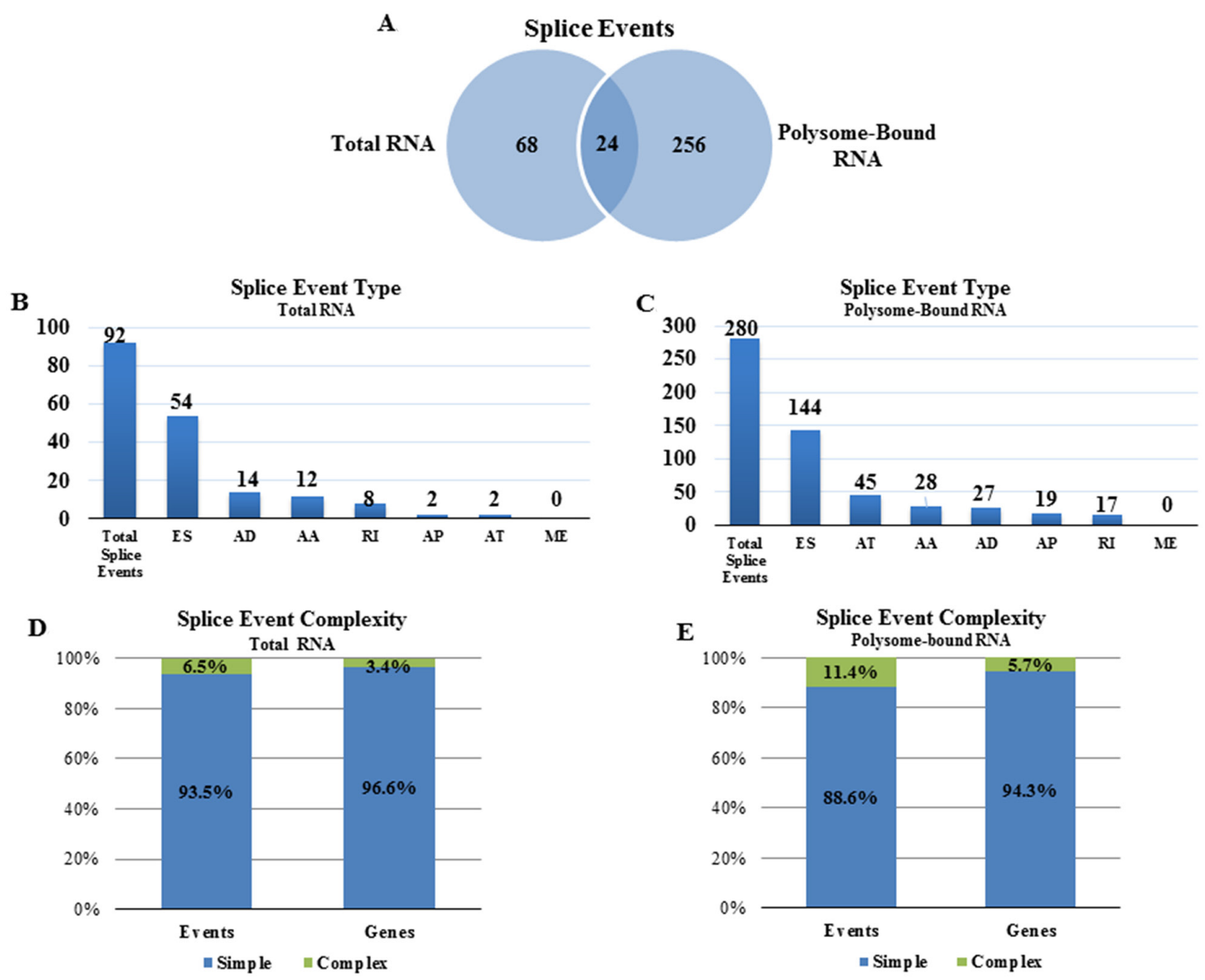

Figure 2: Radiation-induced alternative splice events in the transcriptome and the translatome. (A) Venn diagram comparing the number of radiation-induced alternative splice events in total versus polysome-bound RNA. Number of radiation-induced splice events in (B) total RNA and (C) polysome-bound RNA according to category as defined in Figure 1. Complexity of radiation-induced splice events in the (D) transcriptome and (E) translatome. Simple (blue) refers to 1 event and complex (green) refers to more than 1 event. 
fibroblasts [21] and peripheral blood mononuclear cells [20] using exon specific microarray platforms. These studies, conducted on the transcriptome (total cellular mRNA), identified a number of genes (depending on the splicing algorithm applied) subject to radiation-induced alternative splicing. As shown here, transcriptome analysis using RNA-Seq/SpliceSeq identified 92 splice events corresponding to 89 genes at $1 \mathrm{~h}$ after irradiation of NSC11 cells. Whereas a quantitative comparison to previous studies is not feasible given the different methods, cell types and irradiation protocols, the data presented here further support the induction of AS of pre-mRNA by ionizing radiation.

A
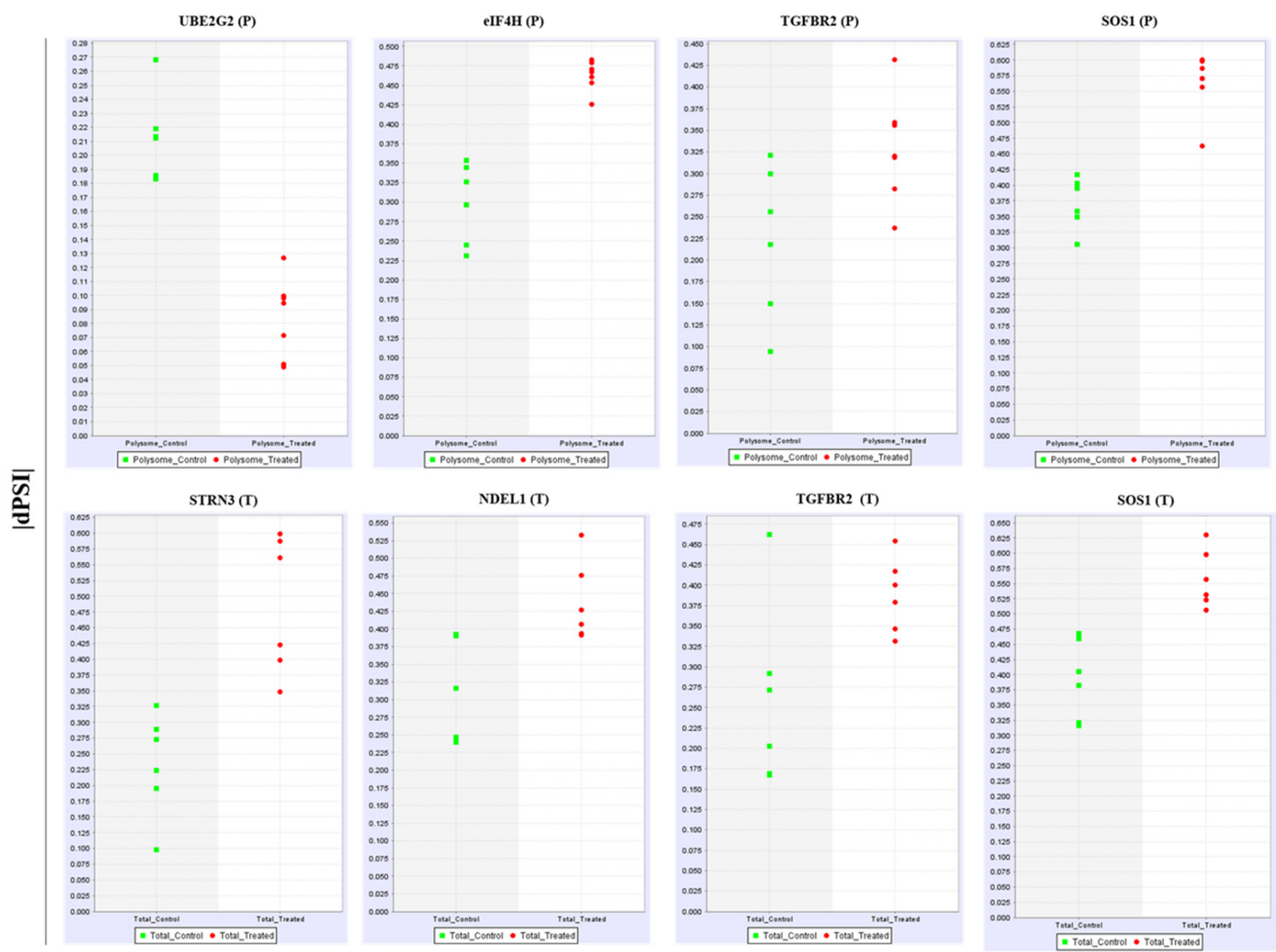

B

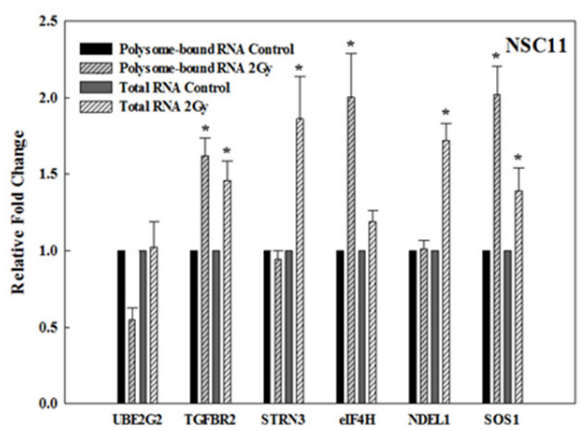

C.

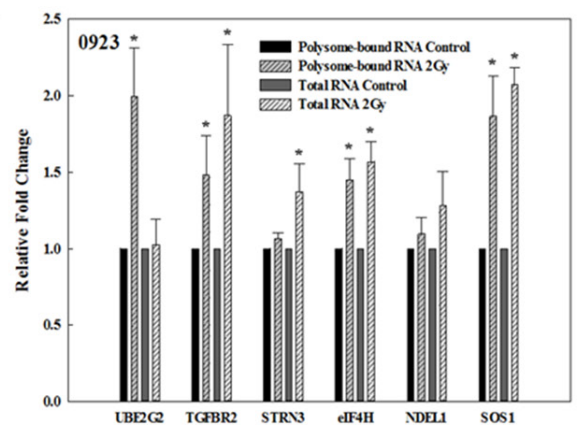

Figure 3: Validation of radiation-induced alternative transcripts in total and polysome-associated RNA. (A) dPSI (change in percent spliced in) scatter plots for the individual replicates of mock irradiated (control, green) and irradiated (2 Gy, $1 \mathrm{~h}$, red) in polysome-associated RNA (P) and total RNA (T). qRT-PCR of polysome-bound RNA and total RNA of the indicated gene normalized to $18 \mathrm{~S}$ rRNA in (B) NSC11 cells and (C) 0923 cells. Each value represents the mean \pm SEM for 3 independent experiments. ${ }^{*} p<0.05$ according to Student's $t$-test (radiation vs. control). 
In addition to using SpliceSeq to identify alternative transcripts, RNA-Seq results were analyzed using the sRAP package to define differential gene expression, i.e., changes in transcript abundance. Comparison of these 2 parameters within the radiation-induced transcriptome indicated that the vast majority of alternative transcripts detected (Figure 5) did not correspond to genes whose expression was modified, in contrast to the data generated using exon arrays $[20,21]$. These results suggest that, at least in NSC11 cells at $1 \mathrm{~h}$ after exposure to $2 \mathrm{~Gy}$, radiation-induced AS and radiation-induced gene expression as determined in total cellular mRNA are independent events. Of note, at $1 \mathrm{~h}$ after irradiation of NSC11 cells most of the radiationinduced changes in the translatome have occurred, whereas the changes in the transcriptome occur at later time points such as $6 \mathrm{~h}[6,8,9]$. Thus, evaluating later times points after irradiation of NSC11 cells may reveal additional AS events within the transcriptome that as shown in previous studies may participate in the DNA damage response [2022]. As eluded to earlier, the rationale for evaluating AS events at the early time point of $1 \mathrm{~h}$ after irradiation was that it reduced the complicating influence of transcriptional changes and allowed for a more direct investigation of the role of AS in radiation-induced translational control of gene expression. Of note, more than half of the AS events detected in both the radiation-induced transcriptome and the radiation-induced translatome were exon skip events, which have been implicated as a source of protein diversity and thus may reflect a change in protein function [13].

Whereas radiation-induced changes in the transcriptome may provide an indicator of radiation exposure $[20,21]$, it is the radiation-induced translatome that corresponds to changes in protein levels playing a functional role in cellular radioresponse [9]. Thus, given the significance of translational control in radiationinduced gene expression along with reports suggesting that

$\mathbf{A}$

Total RNA

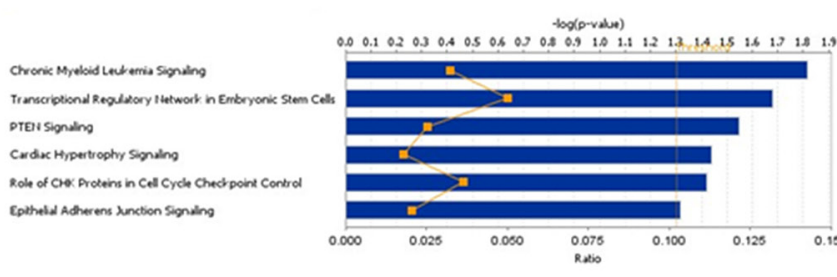

\section{Polysome-Bound RNA}

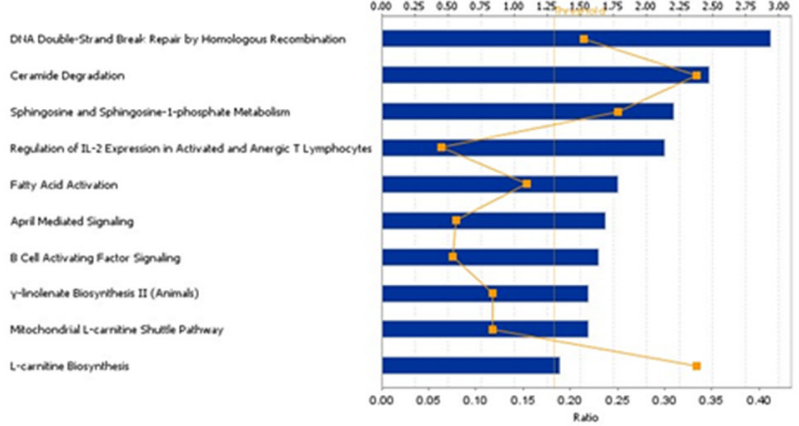

B

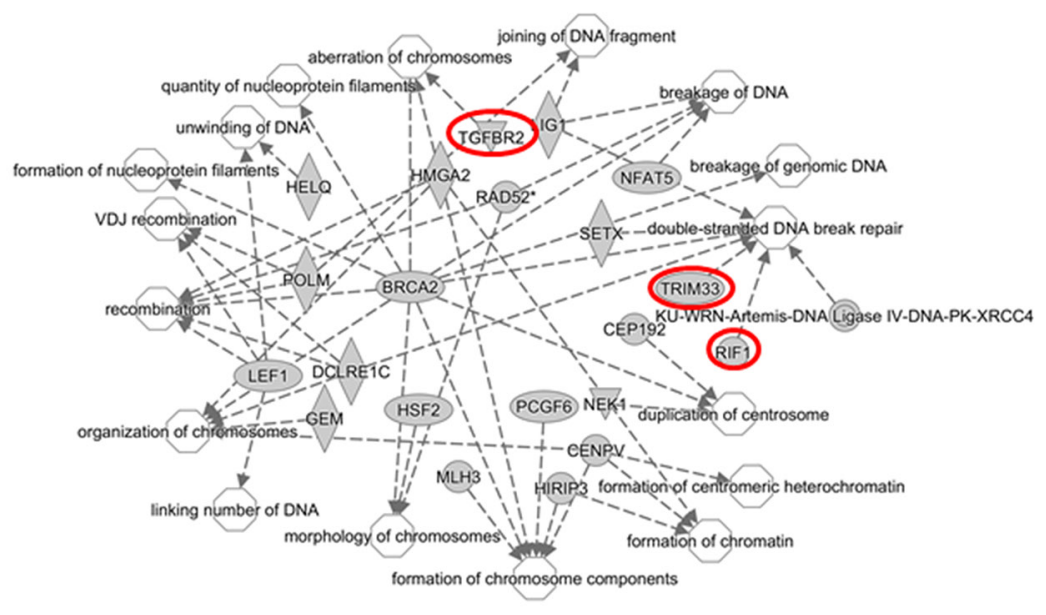

Figure 4: Functional analyses of radiation-induced alternative transcripts. (A) Canonical pathways as defined by IPA for genes associated with radiation-induced changes in alternative transcripts in total RNA and polysome-bound RNA. Bars correspond to $-\log (p$-value) of the pathway's enrichment. The threshold (dotted orange line) is set by IPA at $p<0.05$; the $\mathrm{p}$-value of $<0.01$ equals $-\log (\mathrm{p}$ value) of 2 . The orange squares indicate the ratio of the number of genes within the pathway affected by radiation out of the total number of genes in the pathway. (B) Network analysis of the function DNA Replication, Recombination, and Repair showing 22 genes within the network (grey symbols) and the associated 17 subnetworks (unfilled symbols). Symbol shape represent the function of the protein (ellipses, transcriptional regulators; diamonds, enzymes; concentric circles, complexes; triangles, kinases; circles, other). Red highlighted genes are also in the Total RNA group. 
Table 1: Top networks (IPA) enriched in the radiation induced alternative splice events in total $R \mathbf{R A}\left[\mathrm{score}=-\log _{10}\right.$ (p-value)]

\begin{tabular}{ll}
\hline Score & \multicolumn{1}{c}{ Top Networks } \\
\hline 40 & Cell-To-Cell Signaling and Interaction, Hematological System Development and Function, Inflammatory Response \\
30 & Developmental Disorder, Hereditary Disorder, Metabolic Disease \\
28 & Cell Signaling, Developmental Disorder, Hereditary Disorder \\
25 & Cancer, Endocrine System Disorders, Gastrointestinal Disease \\
24 & Cell-To-Cell Signaling and Interaction, Inflammatory Response, Cellular Growth and Proliferation \\
23 & Cell Morphology, Cellular Function and Maintenance \\
16 & Amino Acid Metabolism, Molecular Transport, Small Molecule Biochemistry \\
12 & Cell Morphology, Cellular Function and Maintenance, Cellular Compromise \\
2 & Cancer
\end{tabular}

Table 2: Top networks (IPA) enriched in the radiation-induced alternative splice events in polysome-bound RNA [score $\left.=-\log _{10}(p-v a l u e)\right]$

\begin{tabular}{|c|c|}
\hline Score & Top Networks \\
\hline 50 & Cell Morphology, Cellular Assembly and Organization, RNA Post-Transcriptional Modification \\
\hline 37 & Cancer, Gastrointestinal Disease, Neurological Disease \\
\hline 35 & Auditory Disease, Cell Morphology, Cellular Function and Maintenance \\
\hline 32 & Cell Morphology, Ophthalmic Disease, Connective Tissue Development and Function \\
\hline 25 & Lipid Metabolism, Small Molecule Biochemistry, Hereditary Disorder \\
\hline 25 & Post-Translational Modification, Developmental Disorder, Hereditary Disorder \\
\hline 23 & Cellular Assembly and Organization, Cellular Function and Maintenance, Developmental Disorder \\
\hline 22 & Cellular Growth and Proliferation, Connective Tissue Development and Function, Cell Cycle \\
\hline 21 & Cellular Movement, Connective Tissue Disorders, Developmental Disorder \\
\hline 21 & DNA Replication, Recombination, and Repair, Energy Production, Nucleic Acid Metabolism \\
\hline 21 & Cell Morphology, Organ Morphology, Reproductive System Development and Function \\
\hline 19 & Cell Death and Survival, Cellular Compromise, Organ Morphology \\
\hline 18 & Cellular Growth and Proliferation, Cell Cycle, Cell-To-Cell Signaling and Interaction \\
\hline 17 & Cell Cycle, Tissue Morphology, Cellular Assembly and Organization \\
\hline 17 & Auditory Disease, Hereditary Disorder, Neurological Disease \\
\hline 17 & Molecular Transport, Small Molecule Biochemistry, Gene Expression \\
\hline 15 & Cancer, Developmental Disorder, Hereditary Disorder \\
\hline 15 & Cell-To-Cell Signaling and Interaction, Metabolic Disease, Cardiac Enlargement \\
\hline 7 & Humoral Immune Response, Protein Synthesis, Cancer \\
\hline 6 & Hematological System Development and Function, Tissue Morphology, Cell-To-Cell Signaling and Interaction \\
\hline 2 & Cancer, Organismal Injury and Abnormalities, Reproductive System Disease \\
\hline 2 & Neurological Disease, Hematological System Development and Function, Organismal Development \\
\hline
\end{tabular}

AS influences translational control [25-27], we defined radiation-induced splicing events in polysome-bound RNA. As compared to the transcriptome, substantially more radiation-induced splice events were identified in the translatome and, importantly, the vast majority were unique to the translatome (Figure 2A). This comparison suggests that the AS of pre-mRNA induced by radiation as detected in the transcriptome does not play a significant role in translational control of gene expression but that radiation modifies the polysome association of existing 
Table 3: Top networks (IPA) enriched in the radiation induced gene expression in total $\mathbf{R N A}\left[\operatorname{score}=-\log _{10}(\mathrm{p}-\mathrm{value})\right]$

\begin{tabular}{|c|c|}
\hline Score & Top Networks \\
\hline 39 & Cell Death and Survival, Nervous System Development and Function, Tissue Morphology \\
\hline 32 & Cell Cycle, DNA Replication, Recombination, and Repair, Organismal Development \\
\hline 27 & Cell Morphology, Organ Morphology, Skeletal and Muscular System Development and Function \\
\hline 26 & Cell-To-Cell Signaling and Interaction, Inflammatory Response, Cellular Function and Maintenance \\
\hline 26 & Nervous System Development and Function, Cell Death and Survival, Cellular Compromise \\
\hline 20 & Reproductive System Development and Function, Nucleic Acid Metabolism, Small Molecule Biochemistry \\
\hline 20 & Auditory and Vestibular System Development and Function, Embryonic Development, Organ Development \\
\hline 19 & Cell-To-Cell Signaling and Interaction, Cellular Compromise, Cellular Function and Maintenance \\
\hline 17 & Cell Death and Survival, Gene Expression, Cell-To-Cell Signaling and Interaction \\
\hline 13 & Hematological System Development and Function, Hypersensitivity Response, Tissue Morphology \\
\hline 11 & Hereditary Disorder, Nervous System Development and Function, Neurological Disease \\
\hline 2 & Developmental Disorder, Hereditary Disorder, Neurological Disease \\
\hline 2 & Cancer, Neurological Disease, Organismal Injury and Abnormalities \\
\hline 2 & Lipid Metabolism, Small Molecule Biochemistry, Vitamin and Mineral Metabolism \\
\hline 2 & Endocrine System Disorders, Hereditary Disorder, Organismal Injury and Abnormalities \\
\hline 2 & Reproductive System Development and Function, Cellular Movement, Post-Translational Modification \\
\hline 2 & Cancer, Endocrine System Disorders, Organismal Injury and Abnormalities \\
\hline 2 & Developmental Disorder, Hereditary Disorder, Ophthalmic Disease \\
\hline
\end{tabular}

alternative transcripts. Functional analysis of these alternative transcripts with respect to cellular processes and pathways showed that the radiation-induced changes detected in polysome bound RNA were enriched with genes corresponding to DNA repair and other aspects of the DNA damage response (Figure 4, Table 2). These results suggest that, in contrast to the alternative transcripts in the radiation-induced transcriptome for which there were no pathways (Figure 4) and few networks (Table 1) detected relevant to radioresponse, the alternate transcripts in the radiation-induced translatome were associated with processes that can influence cell survival.

An additional difference between the alternative transcripts in the radiation-induced transcriptome and translatome involved the relationship to gene expression. In contrast to the transcriptome, a substantial percentage

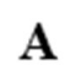

A

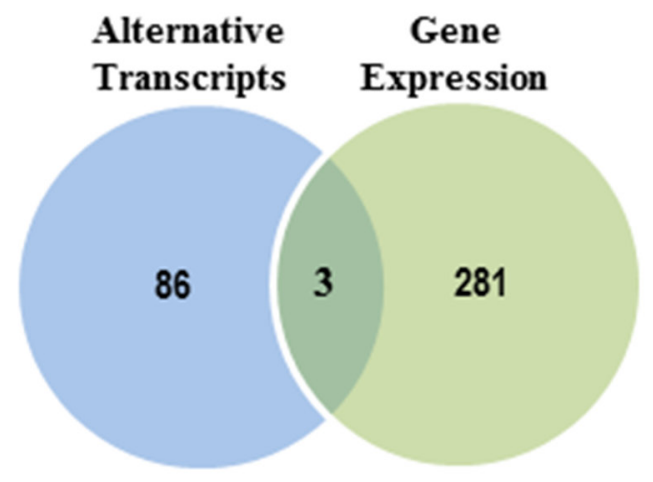

B Polysome-Bound RNA

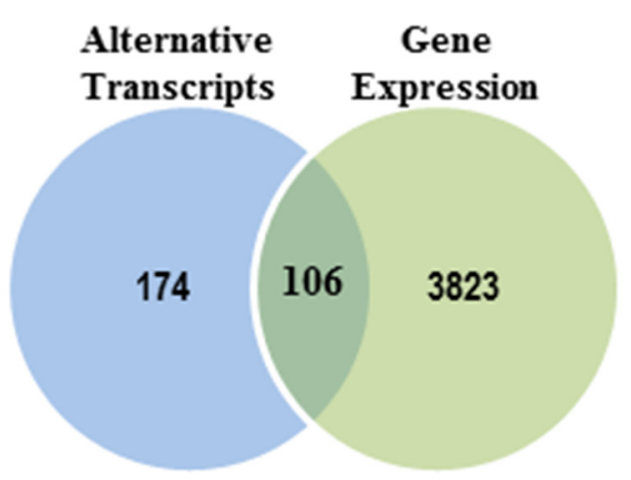

Figure 5: Radiation-induced splice events versus radiation-induced changes in gene expression. Venn diagrams comparing the genes with significant splice events after radiation and genes whose overall abundance (expression) was significantly changed after radiation in (A) total RNA and (B) polysome-bound RNA. 
Table 4: Top networks (IPA) enriched in the radiation induced gene expression in polysome-bound RNA [score $=$ $\left.-\log _{10}(p-v a l u e)\right]$

\begin{tabular}{|c|c|}
\hline Score & Top Diseases and Functions \\
\hline 45 & Developmental Disorder, Hereditary Disorder, Metabolic Disease \\
\hline 43 & Auditory Disease, Developmental Disorder, Endocrine System Disorders \\
\hline 43 & Cellular Assembly and Organization, DNA Replication, Recombination, and Repair, Developmental Disorder \\
\hline 40 & Cellular Assembly and Organization, Cellular Function and Maintenance, Cell Cycle \\
\hline 40 & Cell Cycle, DNA Replication, Recombination, and Repair, Reproductive System Development and Function \\
\hline 38 & Cardiovascular Disease, Developmental Disorder, Hematological Disease \\
\hline 38 & Cellular Function and Maintenance, Nervous System Development and Function, Cell Death and Survival \\
\hline 38 & Post-Translational Modification, Protein Folding, Lipid Metabolism \\
\hline 37 & Drug Metabolism, Endocrine System Development and Function, Lipid Metabolism \\
\hline 36 & Energy Production, Nucleic Acid Metabolism, Small Molecule Biochemistry \\
\hline 34 & Cancer, Hematological Disease, Immunological Disease \\
\hline 32 & Cellular Assembly and Organization, Developmental Disorder, Hereditary Disorder \\
\hline 32 & RNA Post-Transcriptional Modification, Inflammatory Response, Cell Cycle \\
\hline 32 & Developmental Disorder, Hereditary Disorder, Metabolic Disease \\
\hline 30 & Auditory Disease, Cardiovascular System Development and Function, Cell-To-Cell Signaling and Interaction \\
\hline 30 & Hereditary Disorder, Neurological Disease, Psychological Disorders \\
\hline 28 & Post-Translational Modification, DNA Replication, Recombination, and Repair, Nucleic Acid Metabolism \\
\hline 28 & Developmental Disorder, Endocrine System Disorders, Hereditary Disorder \\
\hline 26 & Dermatological Diseases and Conditions, Developmental Disorder, Hereditary Disorder \\
\hline 26 & Developmental Disorder, Neurological Disease, Organismal Injury and Abnormalities \\
\hline 26 & Molecular Transport, RNA Trafficking, Cell Cycle \\
\hline 26 & Embryonic Development, Organ Development, Organ Morphology \\
\hline 25 & Cell Cycle, Cellular Assembly and Organization, Cellular Function and Maintenance \\
\hline 25 & Cellular Development, Cellular Function and Maintenance, Cellular Growth and Proliferation \\
\hline 24 & Gene Expression, Protein Synthesis, Energy Production \\
\hline 23 & Drug Metabolism, Protein Synthesis, Free Radical Scavenging \\
\hline 23 & Cellular Assembly and Organization, Cellular Function and Maintenance, Cell Signaling \\
\hline 21 & Cellular Development, Cell Cycle, Connective Tissue Development and Function \\
\hline 21 & Molecular Transport, Cell Death and Survival, Cellular Compromise \\
\hline 21 & Cancer, Hematological Disease, Organismal Injury and Abnormalities \\
\hline 20 & Respiratory Disease, Organismal Injury and Abnormalities, Renal and Urological Disease \\
\hline 19 & Developmental Disorder, Hereditary Disorder, Metabolic Disease \\
\hline 18 & Cell Death and Survival, Carbohydrate Metabolism, Lipid Metabolism \\
\hline 18 & Gene Expression, Cellular Development, Cancer \\
\hline 18 & Cell Morphology, Cell Cycle, Nervous System Development and Function \\
\hline 18 & Connective Tissue Disorders, Developmental Disorder, Hematological Disease \\
\hline 17 & Cell-To-Cell Signaling and Interaction, Cancer, Organismal Injury and Abnormalities \\
\hline 17 & Tissue Morphology, Metabolic Disease, Cancer \\
\hline 17 & Behavior, Cardiovascular System Development and Function, Organ Morphology \\
\hline 15 & Cellular Development, Hematological System Development and Function, Hematopoiesis \\
\hline
\end{tabular}


of the alternative transcripts detected in the radiationinduced translatome corresponded to genes whose expression was also modified, which suggests that the selective polysome association of the existing alternative transcripts has a quantitative effect on radiation-induced gene translation. The alternative transcripts in the radiation-induced translatome that do not overlap with gene expression, while perhaps not affecting translational efficiency, could qualitatively influence other aspects of protein function such as location and activity. With respect to the mechanism through which alternative transcripts are recruited to and away from polysomes, Sterne-Weiler et al. proposed that AS not only provides a process for increasing protein diversity, but also modifies the cisregulatory structure of mRNAs resulting in an altered susceptibility to post-transcriptional regulatory processes [15]. This scenario could be applicable to the posttranscriptional operon model of Keene and Tenenbaum [28], which proposes that RNA-binding proteins and microRNAs regulate the translation of functionally related genes. Extending these hypotheses to irradiated cells, the AS events operative in untreated NSC11 cells produce a percentage of alternative transcripts for a given gene that have a structural modification that influences its interaction with a radiation-induced RNA-binding proteins (RBP) or microRNA, which then ultimately affects its translation initiation and polysome association. Futhermore, it has been shown that long non-coding RNAs (lncRNAs) also associate with ribosomes and may contribute to the radiation-induced translatome [29]. With respect to specific signaling molecules, ATM, an apical kinase in the radiation-induced DNA damage response, has been implicated in splicesome regulation suggesting a possible role in radiation-induced AS detected in the transcriptome [30]. Whether it plays a role in the radiation-induced recruitment of mRNA isoforms remains to be determined. Clearly, further investigations into the processes through which AS influences translation initiation are required.

The data presented here suggest that whereas radiation induces $\mathrm{AS}$, it is the alternative transcripts present at the time of irradiation that may play a role radiation-induced translational control of gene expression and thus cellular radioresponse. Although the studies described here were conducted using a single human tumor cell line, given that radiation-induced translational control of gene expression is a fundamental component of cellular radioresponse, and that many of the changes in isoforms were also observed in another GSC line, the selective modification of the polysome-binding of existing alternative transcripts is likely to be a common response to radiation. This is supported by the data in Figure 3 showing similar radiation-induced splicing events in another GSC line. Whether there is a difference between tumor and normal cells, as previously shown for radiation-induced translatomes [24] remains to be determined. However, aberrant splicing of premRNA has been implicated in oncogenesis and tumor cell proliferation [31, 32]. Moreover, modulating AS through inhibition of spliceosome activity has been suggested as a cancer treatment strategy [33] with the spliceosome inhibitor E7107 entered into clinical trials [34]. Although more investigation is required, the data presented here indicates the AS plays a role in radiationinduced translational control of gene expression and suggests that the spliceosome may also provide a target for radiosensensitization.

\section{MATERIALS AND METHODS}

\section{Cell lines and treatments}

Studies were performed using the glioblastoma stem-like cell (GSC) lines NSC11 (kindly provided by Dr. Frederick Lang, MD Anderson Cancer Center) and 0923 [35], which were generated from a glioblastoma surgical specimens as previously described [36]. Cell lines were revived every 2 months from frozen stocks made after receiving cell lines and were most recently authenticated in May 2015, by STR analysis (Idexx Laboratories). Neurospheres were maintained in stem cell medium consisting of DMEM/F12 (Invitrogen), B27 supplement (Invitrogen), and human recombinant bFGF and EGF (50 ng/ml each, R\&D Systems) at $37^{\circ} \mathrm{C}$, $5 \% \mathrm{CO}_{2} / 5 \% \mathrm{O}_{2}$. CD133+ NSC11 cells were isolated from neurosphere cultures by FACS and used as a source for the described experiments [37]. The CD133+ cell cultures met the criteria for tumor stem-like cells including selfrenewal, differentiation along glial and neuronal pathways, expression of stem cell related genes, and formation of brain tumors when implanted in immunodeficient mice. For use in an experiment, CD133+ neurosphere cultures were disaggregated into single cells as described [37] and seeded onto poly-L-ornithine (Invitrogen)/laminin (Sigma) coated tissue culture dishes in stem cell media. Under these conditions, single-cell GSCs attach and proliferate maintaining their CD133+ expression and stem-like characteristics [38]. All cells were cultured for less than 2 months after resuscitation. Radiation was delivered using a $320 \mathrm{kV}$ X-ray machine (Precision X Ray Inc.) at a dose rate of $2.3 \mathrm{~Gy} / \mathrm{min}$; control cultures were mock irradiated.

\section{Polysome isolation}

Isolation of polysome-bound RNA was performed on 6 biological replicates initiated from different frozen stocks following the procedure described by Galban et al [39] with slight modifications. Briefly, cells were grown to $\sim 80 \%$ confluency in $150-\mathrm{mm}^{2}$ culture dishes and irradiated ( 2 Gy) or mock irradiated. One hour after irradiation cells were incubated with $100 \mu \mathrm{g} / \mathrm{ml}$ of cycloheximide for 15 minutes at $37^{\circ} \mathrm{C}, 5 \% \mathrm{CO}_{2} / 5 \% \mathrm{O}_{2}$. Cytoplasmic RNA was collected 
by lysing cells in polysome buffer $[15 \mathrm{mmol} / \mathrm{L}$ Tris- $\mathrm{HCl}$ (pH 7.5), $300 \mathrm{mmol} / \mathrm{L} \mathrm{NaCl}, 15 \mathrm{mmol} / \mathrm{L} \mathrm{MgCl} 2,1 \%$ Triton $\mathrm{X} 100,100 \mu \mathrm{g} / \mathrm{mL}$ cycloheximide, $1 \mathrm{mg} / \mathrm{mL}$ heparin, and 500 units/mL RNasin (Promega)]. After 15 minutes on ice, lysates were centrifuged $(12,000 \times \mathrm{g}$ for 15 minutes $)$, and the resulting cytosolic supernatant was layered onto a $10 \%$ to $50 \%$ sucrose gradient. Gradients were then centrifuged at $35,000 \times \mathrm{g}$ for 3 hours at $4^{\circ} \mathrm{C}$ and polysome-bound fractions were collected using an ISCO Density Gradient Fractionation System (ISCO, Lincoln, NE) with continuous monitoring based on A254. Polysome-bound RNA was collected corresponding to pooled fractions 4-12 obtained from sucrose gradient fractionation [9] and extracted using TRIzol LS (Invitrogen). The integrity of the RNA was assured using a Bioanalyzer (Agilent).

\section{Total RNA isolation}

Total RNA, from 6 biological replicates corresponding to the same replicates of polysome-bound RNA, was extracted from irradiated (2 Gy) or mock irradiated cells using TRIzol (Invitrogen) followed by the RNeasy Mini Kit (Qiagen). The integrity of the RNA was assured using a Bioanalyzer (Agilent).

\section{RNA-seq}

RNA-Seq was performed by the Center for Cancer Research Sequencing Facility in Frederick, Maryland. Briefly, between $100 \mathrm{ng}$ to $1 \mu \mathrm{g}$ of total RNA was used as the input for mRNA capture with oligo-dT coated magnetic beads. The mRNA was fragmented followed by random-primed cDNA synthesis. The resulting double-strand cDNA was used as the input to a standard Illumina library prep with end-repair, adapter ligation and PCR amplification to generate a sequencing ready library. The final library was then quantitated by qPCR before cluster generation and sequencing on the Illumina HiSeq2500 sequencer. The HiSeq Real Time Analysis software (RTA 1.18) was used for processing image files, the Illumina CASAVA_v1.8.4 was used for demultiplex and converting binary base calls and qualities to fastq format. The sequencing reads were trimmed of adapters and low quality bases using Trimmomatic (version 0.3), the trimmed reads were aligned to human hg19 reference genome (GRCh37/UCSC hg19) and Ensembl annotation version 70 using TopHat_v2.0.8 software. Given the aligned sequencing reads and a list of genomic features, counts of mapped reads for each gene were calculated using HTSeq [40]. (See Supplementary Table 1 and Supplementary Figure 1 for library complexity)

\section{Alternative transcript and gene expression analysis}

The bioinformatics program SpliceSeq, which was developed for alternative splicing analysis of RNA-Seq data [41], was used to identify differential splicing events in polysome-bound RNA and total RNA. SpliceSeq returns gene reads per kilobase of transcript per million aligned reads (RPKM) and percent spliced in (PSI) values for each gene and every potential splice event with sufficient read coverage. If 2 or more reads overlap into adjacent exons by more than 4 bases, it is considered an observed splice junction. The PSI values of 6 irradiated samples were compared to the PSI values of 6 control samples to identify robust splicing events. A splice event was defined as significant according to the following criteria: 1) The event was reproducible in at least 5 of 6 replicates; 2) the gene expression RPKM (measure of general read coverage) $\geq 2 ; 3$ ) the absolute value of the change in percent of all isoforms displaying the splice event (change in percent spliced in, dPSI) was $\geq 0.1$; 4) the proportion of isoforms containing the splice event was $\geq 0.5$, and 5) the $p$-value of a t-test for difference between the test and control PSI values was $\leq 0.05$.

For gene expression analysis, RPKM reads were used for pairwise comparison between untreated control and irradiated groups for polysome-bound RNA or total RNA samples. This analysis was performed in R [42] with the sRAP package analysis pipeline [43]. Briefly, RPKM values were filtered based upon RPKM cutoff of 0.1 (to avoid bias from low-coverage genes as suggested by http://bioinfo.aizeonpublishers.net/content/2013/6/ bioinfo285-292.pdf), and then data is $\log 2$ transformed (so that the data more closely follows a normal distribution [43]. In all cases, p-values are calculated via linear regression of ANOVA, and false-discovery rates (FDR) are calculated [44]. We set the p-value and FDR threshold to 0.05 . Sequence data has been deposited in NCBI's Gene Expression Omnibus [45] and are accessible through GEO Series accession number GSE94693 (http://www.ncbi.nlm. nih.gov/geo/query/acc.cgi?acc=GSE94693).

\section{qPCR of alternative transcripts}

Complementary first-strand DNA was generated from irradiated and mock-irradiated total and polysomebound RNA isolated separately from the RNA used for RNA-Seq, using the Applied Biosystems HighCapacity RNA-to-cDNA Kit (Applied Biosystems, ThermoFisher) according to the manufacturer's protocol. TaqMan Primers and probes for the specified genes (Applied Biosystems, ThermoFisher), selected based on sequence homology to exon flanking regions, were used and PCR was performed using TaqMan RT-PCR kits (Applied Biosystems, ThermoFisher), according to the manufacturer's protocol. All assays (probes and primers) used are listed in Supplementary Table 7. Relative fold 
changes were determined by the $\Delta \Delta \mathrm{C}_{\mathrm{t}}$ method using 18s (Applied Biosystems, ThermoFisher) as an internal control. Statistical significance $(p \leq 0.05)$ was determined using Student's $t$-test with data expressed as the mean \pm standard error.

\section{Ingenuity pathway analysis}

Genes exhibiting significant changes in alternative splicing or gene expression were submitted to Ingenuity Pathway Analysis (IPA ${ }^{\circledR}$, QIAGEN Redwood City, www. qiagen.com/ingenuity) for core expression analysis and evaluated using canonical pathway and network analyses with IPA default settings and a p-value $\leq 0.01$.

\section{Author contributions}

AW participated in the design of the study, carried out molecular studies, data analysis and drafting the manuscript. MCR carried out data analysis and participated in the design of the study. UTS carried out data analysis and participated in the design of the study. $\mathrm{KC}$ participated in the design and coordination of the study and participated in drafting the manuscript. PJT conceived of the study, participated in the design and coordination and drafting the manuscript. All authors read and approved the final manuscript.

\section{CONFLICTS OF INTEREST}

None.

\section{FUNDING}

Division of Basic Sciences, Intramural Program, National Cancer Institute (Z1ABC011372, Z1ABC011373).

\section{Editorial note}

This paper has been accepted based in part on peerreview conducted by another journal and the authors' response and revisions as well as expedited peer-review in Oncotarget.

\section{REFERENCES}

1. Amundson SA, Bittner M, Chen Y, Trent J, Meltzer P, Fornace AJ Jr. Fluorescent cDNA microarray hybridization reveals complexity and heterogeneity of cellular genotoxic stress responses. Oncogene. 1999; 18:3666-72. https://doi. org/10.1038/sj.onc. 1202676.

2. Ding LH, Shingyoji M, Chen F, Hwang JJ, Burma S, Lee C, Cheng JF, Chen DJ. Gene expression profiles of normal human fibroblasts after exposure to ionizing radiation: a comparative study of low and high doses. Radiat Res. 2005; 164:17-26.
3. Guo WF, Lin RX, Huang J, Zhou Z, Yang J, Guo GZ, Wang SQ. Identification of differentially expressed genes contributing to radioresistance in lung cancer cells using microarray analysis. Radiat Res. 2005; 164:27-35.

4. Long XH, Zhao ZQ, He XP, Wang HP, Xu QZ, An J, Bai B, Sui JL, Zhou PK. Dose-dependent expression changes of early response genes to ionizing radiation in human lymphoblastoid cells. Int J Mol Med. 2007; 19:607-15.

5. Otomo T, Hishii M, Arai H, Sato K, Sasai K. Microarray analysis of temporal gene responses to ionizing radiation in two glioblastoma cell lines: up-regulation of DNA repair genes. J Radiat Res. 2004; 45:53-60.

6. Short SC, Buffa FM, Bourne S, Koritzinsky M, Wouters BG, Bentzen SM. Dose- and time-dependent changes in gene expression in human glioma cells after low radiation doses. Radiat Res. 2007; 168:199-208. https://doi. org/10.1667/RR0940.1.

7. Szkanderova S, Port M, Stulik J, Hernychova L, Kasalova I, Van Beuningen D, Abend M. Comparison of the abundance of 10 radiation-induced proteins with their differential gene expression in L929 cells. Int J Radiat Biol. 2003; 79:623-33.

8. Lu X, de la Pena L, Barker C, Camphausen K, Tofilon PJ. Radiation-induced changes in gene expression involve recruitment of existing messenger RNAs to and away from polysomes. Cancer Res. 2006; 66:1052-61. https://doi. org/10.1158/0008-5472.CAN-05-3459.

9. Wahba A, Rath BH, Bisht K, Camphausen K, Tofilon PJ. Polysome profiling links translational control to the radioresponse of glioblastoma stem-like cells. Cancer Res. 2016. https://doi.org/10.1158/0008-5472.CAN-15-3050.

10. Braunschweig U, Gueroussov S, Plocik AM, Graveley BR, Blencowe BJ. Dynamic integration of splicing within gene regulatory pathways. Cell. 2013; 152:1252-69. https://doi. org/10.1016/j.cell.2013.02.034.

11. Castle JC, Zhang C, Shah JK, Kulkarni AV, Kalsotra A, Cooper TA, Johnson JM. Expression of 24,426 human alternative splicing events and predicted cis regulation in 48 tissues and cell lines. Nat Genet. 2008; 40:1416-25. https:// doi.org/10.1038/ng.264.

12. Pan Q, Shai O, Lee LJ, Frey BJ, Blencowe BJ. Deep surveying of alternative splicing complexity in the human transcriptome by high-throughput sequencing. Nat Genet. 2008; 40:1413-5. https://doi.org/10.1038/ng.259.

13. Wang ET, Sandberg R, Luo S, Khrebtukova I, Zhang L, Mayr C, Kingsmore SF, Schroth GP, Burge CB. Alternative isoform regulation in human tissue transcriptomes. Nature. 2008; 456:470-6. https://doi.org/10.1038/nature07509.

14. Ni JZ, Grate L, Donohue JP, Preston C, Nobida N, O’Brien G, Shiue L, Clark TA, Blume JE, Ares M Jr. Ultraconserved elements are associated with homeostatic control of splicing regulators by alternative splicing and nonsense-mediated decay. Genes Dev. 2007; 21:708-18. https://doi.org/10.1101/ gad.1525507.

15. Sterne-Weiler T, Martinez-Nunez RT, Howard JM, Cvitovik I, Katzman S, Tariq MA, Pourmand N, Sanford JR. Frac-seq 
reveals isoform-specific recruitment to polyribosomes. Genome Res. 2013; 23:1615-23. https://doi.org/10.1101/ gr.148585.112.

16. Wong QW, Vaz C, Lee QY, Zhao TY, Luo R, Archer SK, Preiss T, Tanavde V, Vardy LA. Embryonic stem cells exhibit mRNA isoform specific translational regulation. PLoS One. 2016; 11:e0143235. https://doi.org/10.1371/journal.pone.0143235.

17. Chen MS, Higashikubo R, Laszlo A, Roti Roti J. Multiple alternative splicing forms of human RAD17 and their differential response to ionizing radiation. Gene. 2001; 277:145-52.

18. Hashimoto Y, Zhang C, Kawauchi J, Imoto I, Adachi MT, Inazawa J, Amagasa T, Hai T, Kitajima S. An alternatively spliced isoform of transcriptional repressor ATF3 and its induction by stress stimuli. Nucleic Acids Res. 2002; 30:2398-406.

19. Matsumoto A, Onoyama I, Nakayama KI. Expression of mouse Fbxw7 isoforms is regulated in a cell cycle- or p53dependent manner. Biochem Biophys Res Commun. 2006; 350:114-9. https://doi.org/10.1016/j.bbrc.2006.09.003.

20. Macaeva E, Saeys Y, Tabury K, Janssen A, Michaux A, Benotmane MA, De Vos WH, Baatout S, Quintens R. Radiation-induced alternative transcription and splicing events and their applicability to practical biodosimetry. Sci Rep. 2016; 6:19251. https://doi.org/10.1038/srep19251.

21. Sprung CN, Li J, Hovan D, McKay MJ, Forrester HB. Alternative transcript initiation and splicing as a response to DNA damage. PLoS One. 2011; 6:e25758. https://doi. org/10.1371/journal.pone.0025758.

22. Quintens R, Verreet T, Janssen A, Neefs M, Leysen L, Michaux A, Verslegers M, Samari N, Pani G, Verheyde J, Baatout S, Benotmane MA. Identification of novel radiation-induced $\mathrm{p} 53$-dependent transcripts extensively regulated during mouse brain development. Biol Open. 2015; 4:331-44. https://doi.org/10.1242/bio.20149969.

23. Mamane Y, Petroulakis E, Martineau Y, Sato TA, Larsson O, Rajasekhar VK, Sonenberg N. Epigenetic activation of a subset of mRNAs by eIF4E explains its effects on cell proliferation. PLoS One. 2007; 2:e242. https://doi. org/10.1371/journal.pone.0000242.

24. Kumaraswamy S, Chinnaiyan P, Shankavaram UT, Lu $\mathrm{X}$, Camphausen K, Tofilon PJ. Radiation-induced gene translation profiles reveal tumor type and cancer-specific components. Cancer Res. 2008; 68:3819-26. https://doi. org/10.1158/0008-5472.CAN-08-0016.

25. Delestienne N, Wauquier C, Soin R, Dierick JF, Gueydan C, Kruys V. The splicing factor ASF/SF2 is associated with TIA-1-related/TIA-1-containing ribonucleoproteic complexes and contributes to post-transcriptional repression of gene expression. FEBS J. 2010; 277:2496-514. https:// doi.org/10.1111/j.1742-4658.2010.07664.x.

26. Michlewski G, Sanford JR, Caceres JF. The splicing factor SF2/ASF regulates translation initiation by enhancing phosphorylation of 4E-BP1. Mol Cell. 2008; 30:179-89. https://doi.org/10.1016/j.molcel.2008.03.013.
27. Sanford JR, Gray NK, Beckmann K, Caceres JF. A novel role for shuttling SR proteins in mRNA translation. Genes Dev. 2004; 18:755-68. https://doi.org/10.1101/ $\operatorname{gad} .286404$.

28. Keene JD, Tenenbaum SA. Eukaryotic mRNPs may represent posttranscriptional operons. Mol Cell. 2002; 9:1161-7.

29. Carlevaro-Fita J, Rahim A, Guigo R, Vardy LA, Johnson R. Cytoplasmic long noncoding RNAs are frequently bound to and degraded at ribosomes in human cells. RNA. 2016; 22:867-82. https://doi.org/10.1261/rna.053561.115.

30. Tresini M, Warmerdam DO, Kolovos P, Snijder L, Vrouwe MG, Demmers JA, van IWF, Grosveld FG, Medema RH, Hoeijmakers JH, Mullenders LH, Vermeulen W, Marteijn JA. The core spliceosome as target and effector of noncanonical ATM signalling. Nature. 2015; 523:53-8. https:// doi.org/10.1038/nature14512.

31. David CJ, Manley JL. Alternative pre-mRNA splicing regulation in cancer: pathways and programs unhinged. Genes Dev. 2010; 24:2343-64. https://doi.org/10.1101/gad.1973010.

32. Kaida D, Schneider-Poetsch T, Yoshida M. Splicing in oncogenesis and tumor suppression. Cancer Sci. 2012; 103:1611-6. https://doi.org/10.1111/j.1349-7006.2012.02356.x.

33. van Alphen RJ, Wiemer EA, Burger H, Eskens FA. The spliceosome as target for anticancer treatment. Br J Cancer. 2009; 100:228-32. https://doi.org/10.1038/sj.bjc.6604801.

34. Hong DS, Kurzrock R, Naing A, Wheler JJ, Falchook GS, Schiffman JS, Faulkner N, Pilat MJ, O'Brien J, LoRusso P. A phase I, open-label, single-arm, dose-escalation study of E7107, a precursor messenger ribonucleic acid (pre-mRNA) splicesome inhibitor administered intravenously on days 1 and 8 every 21 days to patients with solid tumors. Invest New Drugs. 2014; 32:436-44. https://doi.org/10.1007/ s10637-013-0046-5.

35. Ene CI, Edwards L, Riddick G, Baysan M, Woolard K, Kotliarova S, Lai C, Belova G, Cam M, Walling J, Zhou M, Stevenson H, Kim HS, et al. Histone demethylase Jumonji D3 (JMJD3) as a tumor suppressor by regulating p53 protein nuclear stabilization. PLoS One. 2012; 7:e51407. https://doi.org/10.1371/journal.pone.0051407.

36. Singh SK, Hawkins C, Clarke ID, Squire JA, Bayani J, Hide T, Henkelman RM, Cusimano MD, Dirks PB. Identification of human brain tumour initiating cells. Nature. 2004; 432:396-401. https://doi.org/10.1038/nature03128.

37. McCord AM, Jamal M, Williams ES, Camphausen K, Tofilon PJ. CD133+ glioblastoma stem-like cells are radiosensitive with a defective DNA damage response compared with established cell lines. Clin Cancer Res. 2009; 15:5145-53. https://doi.org/10.1158/1078-0432.CCR-09-0263.

38. Pollard SM, Yoshikawa K, Clarke ID, Danovi D, Stricker S, Russell R, Bayani J, Head R, Lee M, Bernstein M, Squire JA, Smith A, Dirks P. Glioma stem cell lines expanded in adherent culture have tumor-specific phenotypes and are suitable for chemical and genetic screens. Cell Stem Cell. 2009; 4:568-80. https://doi.org/10.1016/j. stem.2009.03.014. 
39. Galban S, Fan J, Martindale JL, Cheadle C, Hoffman B, Woods MP, Temeles G, Brieger J, Decker J, Gorospe M. von Hippel-Lindau protein-mediated repression of tumor necrosis factor alpha translation revealed through use of cDNA arrays. Mol Cell Biol. 2003; 23:2316-28.

40. Anders S, Pyl PT, Huber W. HTSeq--a Python framework to work with high-throughput sequencing data. Bioinformatics. 2015; 31:166-9. https://doi.org/10.1093/bioinformatics/btu638.

41. Ryan MC, Cleland J, Kim R, Wong WC, Weinstein JN. SpliceSeq: a resource for analysis and visualization of RNA-Seq data on alternative splicing and its functional impacts. Bioinformatics. 2012; 28:2385-7. https://doi. org/10.1093/bioinformatics/bts452.
42. Team RC. R: a language and environment for statistical computing. R Foundation for Statistical Computing, Vienna, Austria. 2016.

43. Warden CD. Optimal calculation of RNA-Seq fold-change values. Int J Comput Bioinfo In Silico Model. 2014; 285 92. https://doi.org/10.18129/B9.bioc.sRAP.

44. Benjamini Y, Hochberg Y. Controlling the false discovery rate: a practical and powerful approach to multiple testing. Journal of the Royal Statistical Society. 1995; 57:289-300.

45. Edgar R, Domrachev M, Lash AE. Gene expression omnibus: NCBI gene expression and hybridization array data repository. Nucleic Acids Res. 2002; 30:207-10. 